University of Nebraska - Lincoln

DigitalCommons@University of Nebraska - Lincoln

Timothy J. Gay Publications

Research Papers in Physics and Astronomy

May 1993

\title{
Investigation of spin-orbit effects in the excitation of noble gases by spin-polarized electrons
}

\author{
J. E. Furst \\ University of Missouri-Rolla, Rolla, Missouri \\ W. M. K. P. Wijayaratna \\ University of Missouri-Rolla, Rolla, Missouri \\ D. H. Madison \\ University of Missouri-Rolla, Rolla, Missouri \\ Timothy J. Gay \\ University of Nebraska - Lincoln, tgay1@unl.edu
}

Follow this and additional works at: https://digitalcommons.unl.edu/physicsgay

Part of the Physics Commons

Furst, J. E.; Wijayaratna, W. M. K. P.; Madison, D. H.; and Gay, Timothy J. , "Investigation of spin-orbit effects in the excitation of noble gases by spin-polarized electrons" (1993). Timothy J. Gay Publications. 20.

https://digitalcommons.unl.edu/physicsgay/20

This Article is brought to you for free and open access by the Research Papers in Physics and Astronomy at DigitalCommons@University of Nebraska - Lincoln. It has been accepted for inclusion in Timothy J. Gay Publications by an authorized administrator of DigitalCommons@University of Nebraska - Lincoln. 
Phys. Rev. A 47, 3775 - 3787 (1993)

[Issue 5 - May 1993]

\title{
Investigation of spin-orbit effects in the excitation of noble gases by spin-polarized electrons
}

\author{
J. E. Furst, W. M. K. P. Wijayaratna, D. H. Madison, and T. J. Gay \\ Laboratory for Atomic and Molecular Research and Department of Physics, \\ University of Missouri-Rolla, Rolla, Missouri 65401
}

Received 2 September 1992

We have measured the integrated Stokes parameters of the light emitted following the impact excitation by polarized electrons of the $n p^{5}(n+1) p[5 / 2]_{3}$ states in $\mathrm{Ne}(n=2)$, Ar $(n=3), \operatorname{Kr}(n=4), \mathrm{Xe}(n=5)$, and the $n p^{5}(n+1) p[5 / 2]_{2}$ state in $\mathrm{Kr}$. The near-threshold linear-polarization fractions $\eta_{1}$ were consistent with zero for all of the $J=3$ states measured, providing no evidence of Mott scattering. For $J=2$ excitation, $\eta_{1}$ shows the clear influence of the intermediately coupled nature of the state. At threshold, the measured circular polarization $\eta_{2}$ and linear polarization $\eta_{3}$ for the $J=3$ states agree well with the $L S$-coupled threshold predictions. These results provide the basis for optical measurements of electron polarization.

C1993 The American Physical Society

URL: http://link.aps.org/abstract/PRA/v47/p3775

DOI: 10.1103/PhysRevA.47.3775

PACS: $34.80 . \mathrm{Nz}, 07.90 .+\mathrm{c}, 13.88 .+\mathrm{e}, 29.27 . \mathrm{Hj}$ 


\title{
Investigation of spin-orbit effects in the excitation of noble gases by spin-polarized electrons
}

\author{
J. E. Furst, ${ }^{*}$ W. M. K. P. Wijayaratna, D. H. Madison, and T. J. Gay \\ Laboratory for Atomic and Molecular Research and Department of Physics, University of Missouri-Rolla, Rolla, Missouri 65401
}

(Received 2 September 1992)

\begin{abstract}
We have measured the integrated Stokes parameters of the light emitted following the impact excitation by polarized electrons of the $n p^{5}(n+1) p[5 / 2]_{3}$ states in $\mathrm{Ne}(n=2), \operatorname{Ar}(n=3), \mathrm{Kr}(n=4), \mathrm{Xe}$ $(n=5)$, and the $n p^{5}(n+1) p[5 / 2]_{2}$ state in $\mathrm{Kr}$. The near-threshold linear-polarization fractions $\eta_{1}$ were consistent with zero for all of the $J=3$ states measured, providing no evidence of Mott scattering. For $J=2$ excitation, $\eta_{1}$ shows the clear influence of the intermediately coupled nature of the state. At threshold, the measured circular polarization $\eta_{2}$ and linear polarization $\eta_{3}$ for the $J=3$ states agree well with the $L S$-coupled threshold predictions. These results provide the basis for optical measurements of electron polarization.
\end{abstract}

PACS number(s): $34.80 . \mathrm{Nz}, 07.90 .+\mathrm{c}, 13.88 .+\mathrm{e}, 29.27 . \mathrm{Hj}$

\section{INTRODUCTION}

The role of spin dependence in electron-atom collisions, as manifested in the exchange and spin-orbit interactions, is obscured in collisions between unpolarized electrons and atoms. However, when polarized electrons are used, these processes may be examined in detail. One type of these measurements involves determination of the Stokes parameters of light emitted by atoms excited by polarized electrons without detecting (integrating) the scattered electron trajectories. It is possible for such experiments to reveal more information about the role of spin processes in the collision than more detailed techniques such as $(e, \gamma e)$ coincidence methods. For example, the Stokes parameter $\eta_{1}$ (defined below) can be shown in some cases to be an unambiguous signature of spin-orbitcoupling effects during the collision in an integrated measurement, whereas in a differential-scattering coincidence experiment, when viewing the photons in a direction perpendicular to the scattering plane, its unique association with such effects is eliminated [1]. A report on what we believe to be the first experiment of this type, designed to look for continuum spin-orbit effects (Mott scattering) using optical techniques, has appeared recently [2]. It showed that, even for a heavy atom such as xenon, continuum spin-orbit effects have negligible influence on the polarization of the emitted radiation. Other integrated Stokes-parameter measurements with polarized electrons have involved either $\mathrm{Hg}$ [3], where the breakdown of $L S$ coupling in the atom and, near threshold, negative-ion resonances, obscure the interpretation of the results, or the alkali metals $[4,5]$, where the emphasis has been on untangling the relative contributions of direct and exchange processes to the total scattering cross section.

In this paper we report our integrated Stokesparameter measurements for collisions between transversely polarized electrons and the heavy noble gases $\mathrm{Ne}$, $\mathrm{Ar}, \mathrm{Kr}$, and $\mathrm{Xe}$. Light emitted along the initial electron polarization direction was observed. We concentrated our efforts on the only excited Russell-Saunders states which are easy to isolate experimentally and which decay optically: the $n p^{5}(n+1) p[5 / 2]_{3}\left({ }^{3} D_{3}\right)$ levels (where $n$ is the principal quantum number of the respective ground states). Studies of these states allow an unambiguous assessment of spin-orbit forces on the continuum electron as a function of target $Z$. To gauge the effect of spinorbit coupling on the target electrons in these collisions, we have also considered departures from $L S$ coupling in the excited states by analyzing photon emission from the $\operatorname{Kr} 5 p^{5} 6 p[5 / 2]_{2}\left({ }^{3} D_{2}\right)$ level as well. In addition to our interest in the basic physics of the collisions, the transitions associated with these states were surveyed to determine their suitability as candidates for optical electron polarimetry. The motivation for the study of these specific states becomes clear upon a review of several relevant theoretical points.

\section{INTEGRATED STATE MULTIPOLES}

The theory for these measurements has been discussed extensively in the past and only those details relevant to the present discussion will be presented in this paper. The integrated Stokes parameters may be expressed as

$$
\begin{aligned}
& \eta_{1}=\frac{I\left(45^{\circ}\right)-I\left(135^{\circ}\right)}{I\left(45^{\circ}\right)+I\left(135^{\circ}\right)}, \\
& \eta_{2}=\frac{I\left(\sigma^{+}\right)-I\left(\sigma^{-}\right)}{I\left(\sigma^{+}\right)+I\left(\sigma^{-}\right)}, \\
& \eta_{3}=\frac{I\left(0^{\circ}\right)-I\left(90^{\circ}\right)}{I\left(0^{\circ}\right)+I\left(90^{\circ}\right)},
\end{aligned}
$$

where $I(\theta)$ is the detected photon intensity with a linearpolarizer transmission axis at an angle $\theta$ with respect to the incident-beam direction $z$ (see Fig. 1) and $\sigma^{+}\left(\sigma^{-}\right)$is the intensity of the positive- (negative-) helicity photons.

The integrated polarization fractions are related to the integrated state multipoles, which are combinations of the excited-state density-matrix elements [6]: 


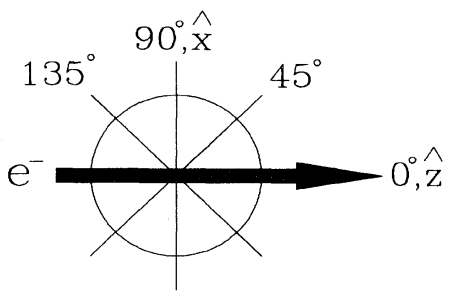

FIG. 1. The collision coordinate system. Electrons are incident along the $z$ axis; the electron-polarization vector is parallel or antiparallel to $\hat{\mathbf{y}}$. The Stokes parameters are defined with respect to the $x-z$ plane for photon emission along $\hat{\mathbf{y}}$.

$$
\begin{aligned}
& I \eta_{1} \propto \operatorname{Re}\left\langle\mathfrak{\Im}_{21}^{\dagger}(J)\right\rangle_{P}, \\
& I \eta_{2} \propto \operatorname{Im}\left\langle\Im_{11}^{\dagger}(J)\right\rangle_{P}, \\
& I \eta_{3} \propto\left\langle\mathfrak{J}_{20}^{\dagger}(J)\right\rangle,
\end{aligned}
$$

where $I$ is the total intensity and the subscript $P$ denotes that only spin-dependent terms contribute to the multipole.

If we assume that the role of the spin-orbit interaction in the collision system is limited to the creation of target fine-structure levels, so that the orbital and spin angular momenta are conserved separately (i.e., $L$ and $S$ of the excited state are good quantum numbers and the continuum electron-nucleus spin-orbit interaction is negligible), then the integrated state multipoles may be expressed by [7]

$$
\begin{aligned}
&\left\langle\Im_{K Q}^{\dagger}(J)\right\rangle=\sum_{\substack{K_{1} Q_{1}, k, q}}(2 k+1)^{1 / 2}\left(2 K_{1}+1\right)^{1 / 2}(2 J+1) \\
& \times\left(K_{1} Q_{1}, k q \mid K Q\right)\left\{\begin{array}{ccc}
K_{1} & k & K \\
L & S & J \\
L & S & J
\end{array}\right\} \\
& \times\left\langle\Im_{K_{1} Q_{1}}^{\dagger}(L)\right\rangle\left\langle T_{k q}^{\dagger}(S)\right\rangle_{P}
\end{aligned}
$$

It should be noted that the assumptions used here may be attributed to Percival and Seaton [8] and do not depend upon any "collision time." The only time scale of importance requires that the fine structure of the excited state have time to evolve before its decay.

Since we are performing an integrated measurement where only the photons are observed, conservation of orbital angular momentum and its projection along a given axis requires that $Q_{1}=0$ [6], so only $\left\langle\Im_{K 0}^{\dagger}(L)\right\rangle$ terms contribute to $\left\langle\mathfrak{\Im}_{K Q}^{\dagger}(J)\right\rangle$. For a well- $L S$-coupled collision system (continuum electron plus atom) the only alignment created during the collision is thus the axial alignment along the $z$ axis, $\left\langle\Im_{20}^{\dagger}(L)\right\rangle$. Moreover, only the orientation created by exchange contributes to the circular polarization, i.e., $\left\langle\mathfrak{\Im}_{11}^{\dagger}(J)\right\rangle_{P}$ is nonzero by virtue of a nonzero $\left\langle T_{11}^{\dagger}(S)\right\rangle_{P}$, since $\left\langle\mathfrak{\Im}_{11}^{\dagger}(L)\right\rangle$ is identically zero for an integrated measurement immediately following the excitation. On the other hand, spin-orbit forces strong enough to affect the total spin of the system, such as those responsible for the breakdown of $L S$ coupling in the excited state or the spin-orbit interaction between the continuum electron and the target, violate the assumptions on which Eq. (3) is based. For either of these processes it is no longer possible to consider $\left\langle\mathfrak{\Im}_{K Q}^{\dagger}(J)\right\rangle$ in terms of factored $L$ and $S$ multipoles:

$$
\left\langle\Im_{K Q}^{\dagger}(L) \times \Im_{k q}^{\dagger}(S)\right\rangle \neq\left\langle\Im_{K Q}^{\dagger}(L)\right\rangle \times\left\langle T_{k q}^{\dagger}(S)\right\rangle_{P},
$$

as is assumed in Eq. (3). In these cases $L$ and $S$ are not conserved separately so that components equivalent to $\mathfrak{\Im}_{K 1}^{\dagger}(L)$ may exist and contribute to the polarization of the light. A careful choice of collision system and excited state makes it possible to untangle the various spindependent processes from each other, using the integrated Stokes parameters. The parameter $\eta_{2}$ may be nonzero due to either exchange or spin-orbit effects. On the other hand, $\eta_{1}$ may only be nonzero if the excited state is not well $L S$ coupled, or, if at some time during the collision, spin-orbit forces play a significant role [6]. This latter case, which we will refer to as "Mott scattering," in analogy with the elastic process, allows the possibility that orbital and spin angular momenta projections along some axis are not conserved separately during the collision. Thus, measuring $\eta_{1}$ allows a probe, solely, of spin-orbit effects.

Another mechanism also exists that may allow a nonzero $\eta_{1}$. A well- $L S$-coupled excited state may be populated via some non-Russell-Saunders intermediate state. An extreme example of this process would be excitation via a non-well- $L S$ coupled negative-ion resonance. If the lifetime of this state is comparable to or longer than its fine-structure relaxation time, $S$ and perhaps $L$ are not good quantum numbers during the protracted collision. This is essentially Mott scattering, i.e., the spin of the "continuum" electron can be rotated by magnetic fields, and $\eta_{1}$ may be nonzero. Such a two-step process might be referred to as a "second-order" process, though it is possible, especially with a narrow resonance, that it might be the dominant mechanism for spin-orbit effects to influence the polarization.

It is possible, even in heavy atoms such as xenon, to have excited states that are well $L S$ coupled. If such an excited state is chosen, any nonzero $\eta_{1}$ is due to the continuum electron-target nucleus interaction and measuring a nonzero $\eta_{1}$ would represent an optical measurement of inelastic Mott scattering [2]. We chose such a state for study so that we could unambiguously identify continuum electron-target spin-orbit effects. The $n p^{5}(n+1) p$ $[5 / 2]_{3}$ level in the noble gases (see Fig. 2) is the only $J=3$ state in the $p$ manifold and is a pure $L S$ state to the extent that configuration mixing (as opposed to intermediate coupling) is unimportant. By contrast, in the $n p^{5}(n+1) p[5 / 2]_{2}$ level, neither $L$ nor $S$ is a good quantum number, even for neon. In this case the wave function can be expressed in an intermediate-coupling representation as

$$
\psi(J=2)=\alpha \psi\left({ }^{3} D_{2}\right)+\beta \psi\left({ }^{1} D_{2}\right)+\delta \psi\left({ }^{3} P_{2}\right),
$$

where $\alpha, \beta$, and $\delta$ are mixing coefficients. A significant change from the $L S$-predicted threshold values of $\eta_{2}$ and 


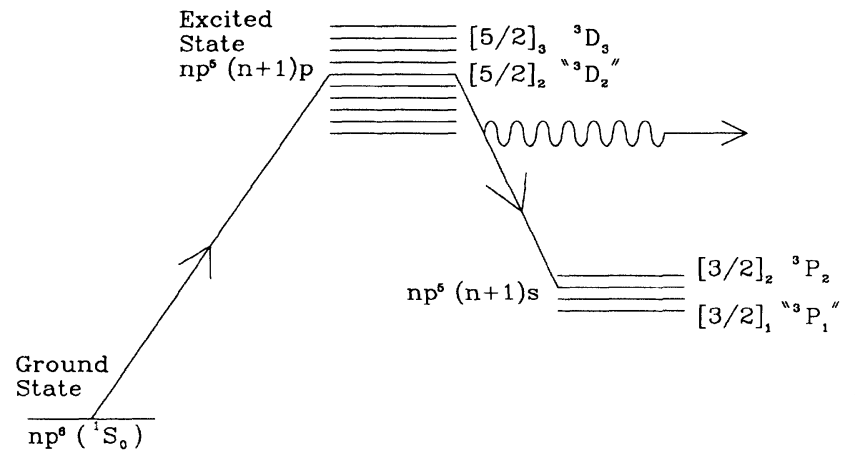

FIG. 2. General energy-level diagram for the noble gases: $\mathrm{Ne}(n=2), \operatorname{Ar}(n=3), \operatorname{Kr}(n=4)$, and $\mathrm{Xe}(n=5)$.

$\eta_{3}$, as well as a nonzero $\eta_{1}$, might be expected since for this state neither $L$ nor $S$ is a good quantum number. Furthermore, if there are no significant spin-orbit forces between the continuum electron and the target nucleus (and this may be demonstrated by measuring $\eta_{1}$ for the $J=3$ state), a nonzero $\eta_{1}$ for the $J=2$ state will be the direct consequence of the breakdown of $L S$ coupling in the excited state.

Table I displays the threshold excitation energies for each of the $J=3$ and $J=2$ states considered, as well as the wavelength of the radiation being measured and the energy and configuration of the nearest level able to cascade into it.

We have calculated the various integrated state multipoles, for pure $L S$ states, in terms of $M_{L}$ cross sections for the ${ }^{3} D_{3}$ and ${ }^{3} D_{2}$ states in the noble gases using Eq. (3). For $L=2$ and $S=1$ with the electrons polarized in the $\hat{\mathbf{y}}$ direction $\left(|\mathbf{P}|=P_{y}\right)$, the only ones that are nonzero if $L S$ coupling holds are

$$
\begin{aligned}
& \left\langle\Im_{11}^{\dagger}(J=3)\right\rangle_{P}=i(0.0831 Q-0.0238 \bar{Q}) P_{y}, \\
& \left\langle\Im_{00}^{\dagger}(J=3)\right\rangle=0.1764 Q, \\
& \left\langle\Im_{20}^{\dagger}(J=3)\right\rangle=0.1746 \bar{Q}, \\
& \left\langle\Im_{11}^{\dagger}(J=2)\right\rangle_{P}=i(0.0248 Q+0.0248 \bar{Q}) P_{y}, \\
& \left\langle\Im_{00}^{\dagger}(J=2)\right\rangle=0.1491 Q, \\
& \left\langle\Im_{20}^{\dagger}(J=2)\right\rangle=0.0891 \bar{Q},
\end{aligned}
$$

where $Q=2 Q_{2}+2 Q_{1}+Q_{0}, \bar{Q}=2 Q_{2}-Q_{1}-Q_{0}$, and $Q_{i}$ is the total cross section for excitation of the $M_{L}=i$ substate. The coefficients preceding $Q$ and $\bar{Q}$ have been calculated using Eq. (3). With these multipoles and Eq. (2) from Eschen et al. [4], the integrated Stokes parameters $\eta_{1}, \eta_{2}$, and $\eta_{3}$ have been determined in terms of the total scattering cross sections $Q_{i}$. The complete analysis is not presented here, but we use it, by considering pure $L S$ coupled states, to predict threshold values of the various polarizations, assuming that at threshold only the cross section $Q_{0}$ is nonzero [8]. In the high-energy limit, longitudinal momentum transfer from the fast electron vanishes and only $Q_{2}$ is nonzero [2]. The parameter $\eta_{1}$ is, of course, zero. These near-threshold and high-energy limiting values are presented in Table II. The differences between the various predictions with $J=3$ are due to the differences in the hyperfine structure of the various isotopes that exist for each gas.

\section{OPTICAL ELECTRON POLARIMETERS}

The idea of optical electron polarimetry was first proposed in detail by Farago and Wykes [9]. When there is excitation by simple exchange, there is a transfer of spin orientation into the target. After the excitation, this spin orientation causes a total angular momentum orientation $\left(J_{y}\right)$ and this orientation causes the emitted photon to have a circular polarization. Farago and Wykes showed that the circular polarization of radiation emitted in a ${ }^{3} S_{1}$ to ${ }^{3} P_{J}$ transition in the group-IIB elements after excitation by polarized electrons is proportional to the initial electron polarization. The exacting experiment of Eminyan and Lampel [10], which used an atomic zinc target, demonstrated the technique, while also establishing the great difficulty in using the group-IIB elements for a useful electron polarimeter. Recently, the $3{ }^{3} P$ to $2{ }^{3} S$ transition in helium has been demonstrated to be effective for electron polarimetry [11], since it overcomes many of the problems that arise when group-IIB targets are used. The relationship between the initial electron polarization and the circular polarization in this case is [12]

$$
\eta_{2}=\left(0.5-\frac{\eta_{3}}{6}\right) P_{y}
$$

The He technique offers the possibility of electron polarization measurements with an accuracy of better than $0.5 \%$ with an ease that makes it vastly superior to the $\mathrm{Zn}$-based device, and in some circumstances even to the standard Mott electron polarimeter. If continuum

TABLE I. Threshold energy and emission wavelengths, together with the energy and $L S$ designation of the nearest cascades for the $n p^{5}(n+1) p[5 / 2]_{J}$ levels in the noble gases.

\begin{tabular}{lccc}
\hline \multicolumn{1}{c}{ Element } & $\begin{array}{c}\text { Threshold } \\
\text { energy }(\mathrm{eV})\end{array}$ & $\begin{array}{c}\text { Emission } \\
\text { wavelength }(\AA)\end{array}$ & $\begin{array}{c}\text { Closest cascades } \\
\text { Energy }(\mathrm{eV})\end{array}$ \\
\hline Xenon $J=3$ & 9.72 & 8819 & $9.94\left(5 p^{5} 5 d^{3} F^{\circ}{ }_{4}\right)$ \\
Krypton $J=3$ & 11.44 & 8112 & $12.11\left(4 p^{5} 4 d\right)$ \\
Argon $J=3$ & 13.07 & 8115 & $14.07\left(3 p^{5} 5 s^{3} P^{\circ}{ }_{2}\right)$ \\
Neon $J=3$ & 18.55 & 6402 & $19.66\left(2 p^{5} 4 s^{3} P^{\circ}{ }_{2}\right)$ \\
Krypton $J=2$ & 11.44 & 8777 & $12.03\left(4 p^{5} 4 d\right)$ \\
\hline \hline
\end{tabular}


TABLE II. Kinematic $L S$-coupling predictions of the threshold and high-energy limits for the polarization fractions $\eta_{2} / P_{e}$ and $\eta_{3}$. At threshold it is assumed that only $M_{L}=0$ states are populated and at high energies that only $\left|\boldsymbol{M}_{L}\right|=2$ states are populated. Cascading is neglected.

\begin{tabular}{lcccc}
\hline \multicolumn{1}{c}{ Element } & Threshold & Threshold & High energy & High energy \\
$\eta_{2} / P_{e}$ & $\eta_{3}$ & $\eta_{2} / P_{e}$ & $\eta_{3}$ \\
\hline Xenon $J=3$ & 0.7080 & 0.3870 & 0.5301 & -0.5215 \\
Krypton $J=3$ & 0.6959 & 0.3997 & 0.5270 & -0.5448 \\
Argon $J=3$ & 0.7317 & 0.4390 & 0.5747 & -0.6207 \\
Neon $J=3$ & 0.7315 & 0.4385 & 0.5743 & -0.5270 \\
Krypton $J=2$ & 0.0000 & 0.3064 & 0.5233 & -0.3850 \\
\hline \hline
\end{tabular}

electron-target spin-orbit forces are negligible, then the excitation of any well- $L S$-coupled state by exchange, and the subsequent analysis of the circular polarization of the radiation emitted, provide information on the initial electron polarization. These conditions may be met in the excitation of the ${ }^{3} D_{3}$ states in the heavy noble gases. These systems have potential advantages over $\mathrm{He} 3{ }^{3} P$ excitation in that excitation cross sections should be higher, yielding more efficient polarimeters. Moreover, for $\mathrm{Ne}$, $\mathrm{Ar}$, and $\mathrm{Kr}$ there is a larger gap between the ${ }^{3} D_{3}$ threshold and the excitation threshold of the next-highest excited state that can cascade to the ${ }^{3} D_{3}$ level, making the input energy and energy width of the electron beam to be analyzed less critical. We have derived expressions, similar to that for $\mathrm{He}$, which relate $\eta_{2}$ to $P_{y}$ in terms of $\eta_{3}$.

When spin-orbit forces are negligible and $\eta_{1}$ is zero, $\eta_{2}$ for triplet states can be expressed in terms of $\eta_{3}$ and $P_{y}$ :

$$
\eta_{2}=3 A\left(1+C \eta_{3}\right) P_{y} .
$$

The coefficients $A$ and $C$ may be derived from Eq. (13) of Bartschat et al. [13], so that

$$
A=\frac{k_{1} a_{1}}{3 k_{2} a_{3}}, \quad B=\frac{k_{1} a_{2}}{k_{3} a_{4}}, \text { and } C=\frac{(B / A)-1}{3} .
$$

The values of $k_{1}, k_{2}$, and $k_{3}$ are

$$
\begin{aligned}
& k_{1}=2\left\{\begin{array}{lll}
1 & 1 & 1 \\
J & J & J_{f}
\end{array}\right\} G_{1}(J), \\
& k_{2}=2 \frac{(-1)^{J+J_{f}}}{3(2 J+1)^{1 / 2}},
\end{aligned}
$$

and

$$
k_{3}=\sqrt{\frac{3}{2}}\left\{\begin{array}{lll}
1 & 1 & 2 \\
J & J & J_{f}
\end{array}\right\} G_{2}(J),
$$

TABLE III. Hyperfine depolarization coefficients $G_{K}(J)$ for the $n p^{5}(n+1) p[5 / 2]_{J}$ levels in the noble gases, corrected for the presence of various isotopic mixtures (see text).

\begin{tabular}{lcc}
\hline \hline \multicolumn{1}{c}{ Element } & $G_{1}(J)$ & $G_{2}(J)$ \\
\hline Xenon $J=3$ & 0.9483 & 0.8639 \\
Krypton $J=3$ & 0.9316 & 0.9116 \\
Argon $J=3$ & 1.0000 & 1.0000 \\
Neon $J=3$ & 0.9995 & 0.9987 \\
Krypton $J=2$ & 0.9275 & 0.9008 \\
\hline \hline
\end{tabular}

where $J\left(J_{f}\right)$ is the total angular momentum of the excited (final) state, the $G_{K}(J)$ 's are the hyperfine depolarization factors [7] corrected for the contributions of the various isotopes [3] (see Table III), and the $a_{i}$ 's are determined from Eq. (5) by

$$
\begin{aligned}
& \left\langle\Im_{11}^{\dagger}(J)\right\rangle_{P}=i\left(a_{1} Q+a_{2} \bar{Q}\right) P_{y}, \\
& \left\langle\Im_{00}^{\dagger}(J)\right\rangle=a_{3} Q,
\end{aligned}
$$

and

$$
\left\langle\Im_{20}^{\dagger}(J)\right\rangle=a_{4} \bar{Q} .
$$

A summary of the polarimeter expressions for all the noble gases, which takes into account the various isotopic and hyperfine mixtures [14], is given in Table IV.

\section{EXPERIMENTAL APPARATUS}

\section{A. Overview}

We measure the polarization of light emitted by raregas atoms after excitation by polarized electrons with energies between 9 and $100 \mathrm{eV}$. The polarized electron beam intersects an effusive atomic beam at right angles and the polarization of photons emitted perpendicularly to both the electron and atomic beam (along the electron spin-polarization direction) is determined. We have concentrated on measurements near threshold, though we present survey measurements for each Stokes parameter up to $100 \mathrm{eV}$. The apparatus comprises three sections: the electron source, a Mott polarimeter, and the target chamber. These are shown schematically in Fig. 3. In Secs. IV B-E we describe briefly the polarized electron source and Mott polarimeter and we describe the optical polarimeter in detail. In Sec. IV F we describe the data acquisition and analysis procedures.

TABLE IV. Electron-polarimeter expressions for the $n p^{5}$ $(n+1) p[5 / 2]_{J}$ levels in the noble gases. These equations give the relationship between $\eta_{2} / P_{e}$ and $\eta_{3}$ for well- $L S$-coupled states in an energy range unaffected by cascades (see text).

\begin{tabular}{lc}
\hline \hline \multicolumn{1}{c}{ Element } & $\eta_{2} / P_{e}$ \\
\hline Xenon $J=3$ & $0.6322\left(1+0.3098 \eta_{3}\right)$ \\
Krypton $J=3$ & $0.6214\left(1+0.2768 \eta_{3}\right)$ \\
Argon $J=3$ & $0.6667\left(1+0.2222 \eta_{3}\right)$ \\
Neon $J=3$ & $0.6663\left(1+0.2230 \eta_{3}\right)$ \\
Krypton $J=2$ & $0.2319\left(1-3.264 \eta_{3}\right)$ \\
\hline \hline
\end{tabular}


POLARIZED

ELECTRON SOURCE

TARGET

CHAMBER

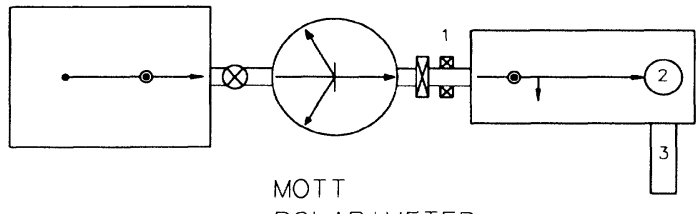

POLAR I METER

FIG. 3. Schematic diagram of the experimental apparatus showing the (1) spin rotator, (2) interaction region, and (3) optical polarimeter.

\section{B. Electron source and Mott polarimeter}

The electron source and Mott polarimeter have been described previously [15]. Briefly, we have a GaAs photoelectron source similar to those described by Pierce et al. [16] and Tang et al. [17]. Longitudinally polarized electrons are emitted after a Cs- and $\mathrm{O}_{2}$-coated GaAs crystal is illuminated with circularly polarized $780-\mathrm{nm}$ radiation. The electron spin is reversed by changing the helicity of the incident radiation with a rotatable quarter-wave plate. Since we require transverse polarization, the spin is rotated by a $90^{\circ}$ electrostatic deflector to give the correct orientation. The source has an emission current of up to $300 \mu \mathrm{A}$ and polarizations that are typically $26-27 \%$, as measured by the Mott polarimeter. We estimate the energy resolution of the electron beam to be about $150 \mathrm{meV}$, comparable to that of similar sources [18]. With the photocathode at $-2000 \mathrm{~V}$, a "stiff" beam is provided for transport through the various valves and the Mott polarimeter before entering the target chamber. The Mott polarimeter is a Farago-Rice concentriccylinder retarding-field device $[19,20]$. Electron polarizations can be measured at analyzing energies up to 125 $\mathrm{keV}$. After the Mott polarimeter and prior to the entrance to the target chamber is a magnetic spin rotator [21] and an electrostatic lens. The spin rotator consists of two 100-turn coils which produce an axial magnetic field able to rotate the electron spin by $90^{\circ}$ in a plane perpendicular to the electron-beam direction, so that the spin is aligned with the axis of the optical polarimeter which is attached to the target chamber. This dual-coil configuration and the electrostatic lens are also used to focus the electron beam into the entrance of the targetchamber electron optics.

\section{Target}

The target chamber consists of an 8-in-diam stainlesssteel tube pumped by two Edwards Diffstak pumps with pumping speeds of 2040 and $6331 / \mathrm{s}$ for air. These

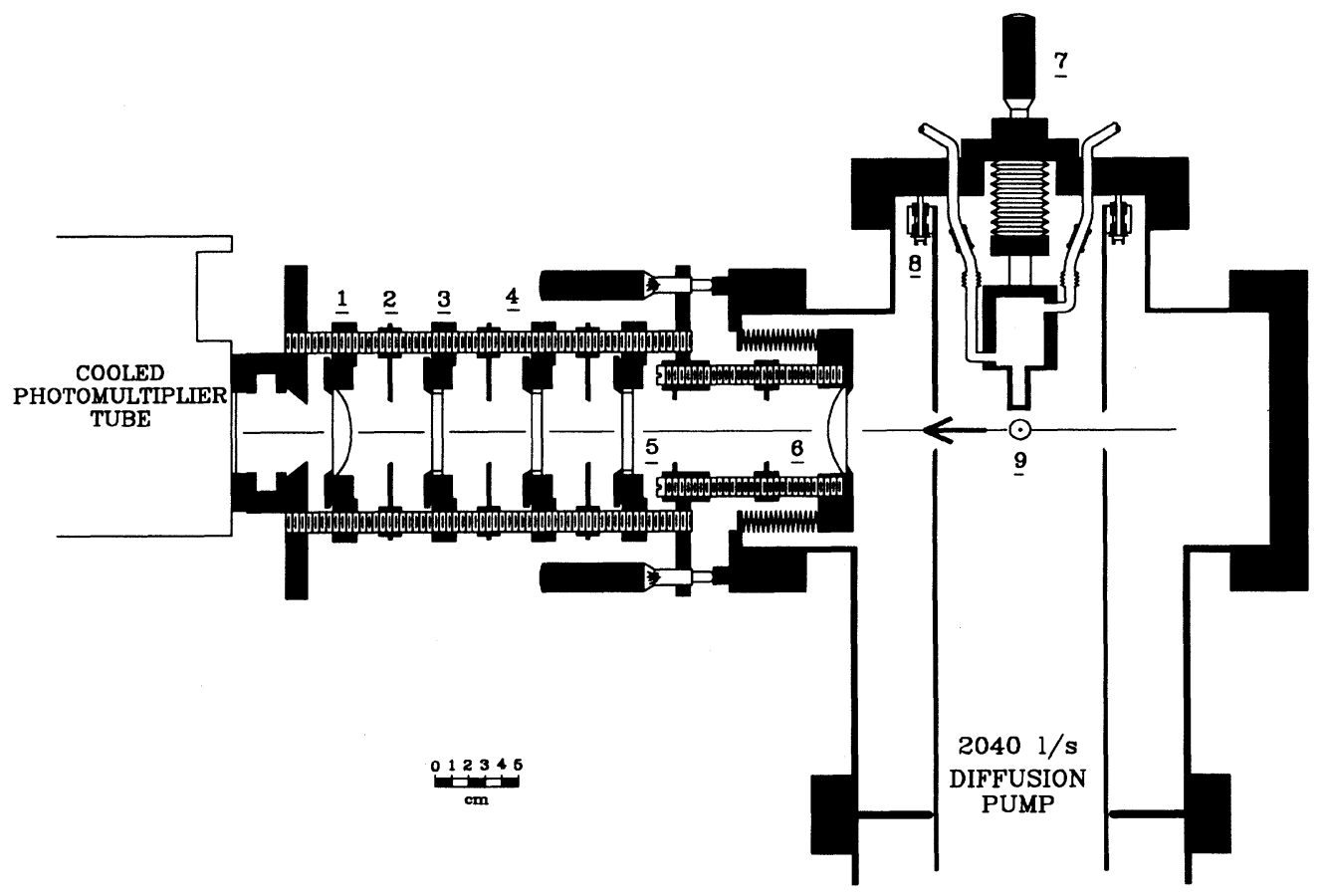

FIG. 4. Interaction region of the target chamber showing the optical polarimeter: (1) refocusing spherical lens; (2) collimator; (3) interference filter; (4) linear polarizer; (5) achromatic retarder; (6) spherical focusing lens on movable bellows; (7) vertically adjustable gas-beam-focusing assembly including capillary array, gas ballast volume, and feed and pressure monitoring lines with ceramic voltage breaks; (8) voltage-floatable cylinder that defines the interaction-region potential and serves as a differential pumping barrier for the target; and (9) interaction region. The electrons are coming out of the page and the electron polarization axis is indicated by the arrow. 
pumps, which include a chilled-methanol cooled $\left(-75^{\circ} \mathrm{C}\right)$ baffle, provide an exceptionally clean vacuum environment with no trace of contamination from either diffusion pump or roughing pump oil at the $10^{-10}$ Torr partialpressure level [22]. This feature is important since the GaAs source lifetime is particularly sensitive to hydrocarbon contamination. The base pressure of the unbaked system is $2 \times 10^{-8}$ Torr. The entire chamber is lined internally with $\mu$-metal magnetic shielding which provides a uniform field of less than $10^{-6} \mathrm{~T}$ in the interaction region.

Our noble-gas target (Fig. 4) is an effusive atomic beam with a density of $\sim 10^{10} \mathrm{~cm}^{-3}$ [23] which is created by flow through a $0.25-\mathrm{cm}$-diam stainless-steel array consisting of $10-\mu \mathrm{m}$-diam channels. Typical pressures in the ballast volume behind the array vary with target gas between 0.7 and 2.5 Torr as measured by a capacitance manometer. The atomic beam is directed into the entrance of the $2040-1 / \mathrm{s}$ pump by a 10.2-cm-diam stainless-steel tube which surrounds the interaction region and provides for differential pumping, allowing high-target pressures with no significant effect on the electron-source stability or lifetime.

\section{Target electron optics}

At the entrance of the target chamber the electron beam is $1 \mathrm{~mm}$ in diameter and has a kinetic energy of $2000 \mathrm{eV}$. It is decelerated to lower energies using a Heddle five-element afocal lens [24] and a final three-element zoom lens. The Heddle lens provides for a deceleration ratio of up to 50:1, changing the energy of the beam from 2000 to $40 \mathrm{eV}$. The final zoom stage is designed to provide a constant beam shape and focus for energies between 10 and $40 \mathrm{eV}$. In the interaction region our beam current is about $1 \mu \mathrm{A}$ at an energy of $10 \mathrm{eV}$. Electrostatic deflectors along the gun allow alignment of the beam. The lens systems in both the target and source chambers are constructed of molybdenum.

Since the GaAs photocathode is at $-2000 \mathrm{~V}$, we define the interaction-region potential by applying a voltage to the differential pumping cylinder surrounding the interaction region. A two-element Faraday cup with a length-to-diameter ratio of 10:1 collects the electron beam. The outer cylinder is maintained at the potential of the last lens element before the interaction region, thereby ensuring a uniform field in the interaction region. There is a bias of $+200 n \mathrm{~V}$, with respect to the potential on the outer cylinder, on the center collector so that no electrons escape the Faraday cup. The current on the center collector is monitored by computer via a generalpurpose-interface-bus- (GPIB) controlled Keithley 485 picoammeter, enabling corrections for changes in electron current during the period of each measurement. The voltages for all the lens elements and the interaction-region cylinder are provided by a potentiometer-controlled voltage divider network that is powered by a stable high-voltage power supply (Fluke 412B). In this configuration, the electron energy is defined by the difference between the photocathode and interaction-region cylinder potentials. With the present configuration there can be long-term drifts of about 0.2
$\mathrm{eV}$ which must be corrected for (see below). The voltage on the final energy-defining lens element and interaction region can also be controlled by a GPIB-programable power supply. This allows the voltage to be adjusted in $0.1-\mathrm{V}$ steps for near-threshold excitation-function measurements.

\section{E. Optical polarimeter}

The optical polarimeter views the interaction region at $90^{\circ}$ to the electron beam (Fig. 4). Its $54-\mathrm{mm}$-diam borosilicate entrance focusing lens (focal length $120 \mathrm{~mm}$ at $545 \mathrm{~nm}$ ) subtends a solid angle of $0.083 \mathrm{sr}$ with an opening half angle of $9.25^{\circ}$. This lens, which also acts as a vacuum window, is mounted on a bellows, permitting adjustment of its position to allow for changes in electronbeam and gas-target alignment as well as the changes in focal length that occur at different wavelengths. The photon beam is further collimated by a series of $38-\mathrm{mm}$ apertures spaced along the optical train, and passes through a combination of polarizer, achromatic retarder, and narrow-band interference filter in appropriate order for each Stokes-parameter measurement. Finally it is refocused onto the photocathode of the photomultiplier by a second borosilicate lens.

The first four optical train elements are arranged in movable, rotatable holders, allowing careful examination of position-dependent instrumental effects. The position of the polarization elements is set by a computercontrolled stepper motor with a precision of better than $0.5^{\circ}$. The principal axis of the polarizer and the fast axis of the retarder are calibrated externally with an accuracy of better than $0.5^{\circ}$.

The extinction factor of the polarizer and the retardance of the achromatic retarder were measured at each wavelength. These values were then used to adjust the measured Stokes-parameter values, giving the true value integrated over the solid angle of acceptance of the detector. The present results are not corrected for solid-angle effects which are estimated to change the value of the Stokes parameters by less than $1 \%$ [25].

Following the polarization elements is a rotatable narrow-band interference filter. The filters we used are centered at the wavelengths indicated in Table I and have a passband of $10 \AA$ (fullwidth at half maximum). A 90$\mathrm{mm}$ focal-length lens is used to focus the light onto the photocathode of a cooled $\left(-27^{\circ} \mathrm{C}\right)$ photomultiplier tube (Hamamatsu R943-02) which has low dark count $\left(<15 \mathrm{~s}^{-1}\right)$ and a quantum efficiency of greater than $7 \%$ for all the wavelengths considered.

For the linear-polarization measurements the polarizer and retarder are rotated together, with the fast axis of the retarder set at $45^{\circ}$ with respect to the polarizer axis. This ensures that only radiation of a fixed helicity passes through the subsequent optics, minimizing instrumental effects. For the circular polarization measurement the retarder is rotated and the polarizer remains fixed.

In the case of the electron-polarization-dependent Stokes parameters $\eta_{1}$ and $\eta_{2}$, instrumental polarizations may be eliminated by reversing the incident electronpolarization direction. The instrumental polarizations 


$$
\left\{\eta_{i}^{I}=\left[\eta_{i}(\text { spin up })+\eta_{i}(\text { spin down })\right] / 2\right\}
$$

were found to be typically 0.02 . The instrumental effects in $\eta_{3}$ should be of the same order though they cannot be eliminated as easily as those for the other two parameters. The measured values of $\eta_{1}$ and $\eta_{2}$ have been corrected for instrumental effects, while those for $\eta_{3}$ have not.

The absolute energy scale was set by determining the voltage at which photons were first detected. This energy corresponds to excitation by electrons in the high-energy "tail" of the electron-energy distribution. Thus our energy scale represents the maximum energy of electrons in our beam which is approximately $75 \mathrm{meV}$ greater than the mean electron energy. Consequently, we can "creep up" on the threshold, thereby giving our experiment an effectively higher energy resolution, enabling us to measure threshold polarizations accurately.

\section{F. Data acquisition and analysis}

We use two methods to make photon polarization measurements. For energies just below the first cascade threshold and above, our final polarization value is the result of a series of measurements that involved counting photons at four polarization analyzer positions for each individual energy. For $\eta_{3}$, measurements were made with the polarizer transmission axis at $0^{\circ}, 90^{\circ}, 180^{\circ}$, and $270^{\circ}$ with respect to the electron-beam direction. For $\eta_{1}$, measurements were made at $45^{\circ}, 135^{\circ}, 225^{\circ}$, and $315^{\circ}$ with respect to the electron-beam direction. Typically, we would accumulate data for $10 \mathrm{~s}$ at each position and do ten cycles during one measurement.

Near threshold, a different technique was used. To minimize the time required at each energy, the analyzer position was set according to the parameter needed and the energy scanned in $0.1-\mathrm{V}$ increments, typically in 32 steps, allowing the accumulation of data at 32 energies. A typical set of spectra with data from two orthogonal polarizer positions is shown in Fig. 5. Thus, for four polarizer positions and 32 energies, the polarizer was moved only four times. Since the rotation of the analyzer occupied a large proportion of the data-acquisition duty cycle, this second technique significantly reduced the acquisition time. Corrections for long-term drifts in energy

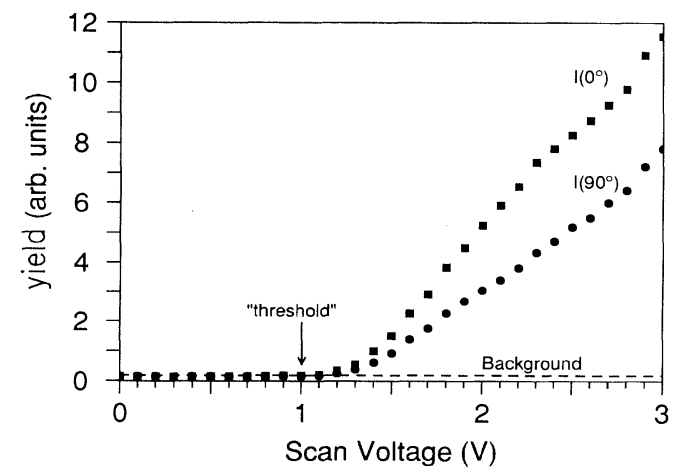

FIG. 5. Typical near-threshold excitation functions $(\mathrm{Kr}$ $J=3$ ) for two linear-polarization fractions. The assigned value of threshold energy is indicated. were made by identifying the threshold voltage for each excitation function and adjusting its corresponding energy scale. In general, all the measurements corresponding to a single set of four polarizer positions had the same voltage at threshold but measurements taken on different days differed in voltage by as much as $0.2 \mathrm{~V}$. Thus the assignment of the correct energy scale for each set was critical for an accurate measurement of the near-threshold polarization. Measurements were made at common energies using each technique to ensure consistent results. In addition, we carefully checked that the threshold energies identified by the onset of photon production and the onset of light polarization were within $0.1 \mathrm{eV}$ of each other.

Several sets of measurements were performed to study possible systematic errors. Of particular concern was any depolarization due to collisions and radiation trapping in the gas beam. We measured each Stokes parameter over a range of beam driving pressures for each gas and found no effect, even at pressures approaching the limit of our pumping capacity. A typical measurement, in this case for $\eta_{2}$ with a neon target at $25 \mathrm{eV}$, is shown in Fig. 6 .

We also checked that our electron-polarization direction corresponded to the axis of our optical polarimeter. We measured $\eta_{2}$, which is proportional to $P_{y}$, as we rotated the electron spin around our incident-beam axis using the magnetic spin rotator and identified the position at which $\eta_{2}$ was maximum. This maximum in $\eta_{2}$ coincides with the polarimeter axis and indicates that the electron spin was aligned correctly.

There were several sources of background that affected the low-count-rate measurements, making near-threshold polarization measurements difficult. Nonelectron-related background came from dark noise in the photomultiplier tube and light leaks in the chamber and optical train. The count rate from both these sources was $<15 \mathrm{~s}^{-1}$ and was primarily due to the dark noise of the tube. This count rate was subtracted from all measurements before any electron-related signal was considered. Electronrelated signal might come from several sources including the following.

(i) Background gas in electron lens elements. These photons exhibit an energy dependence related to the volt-

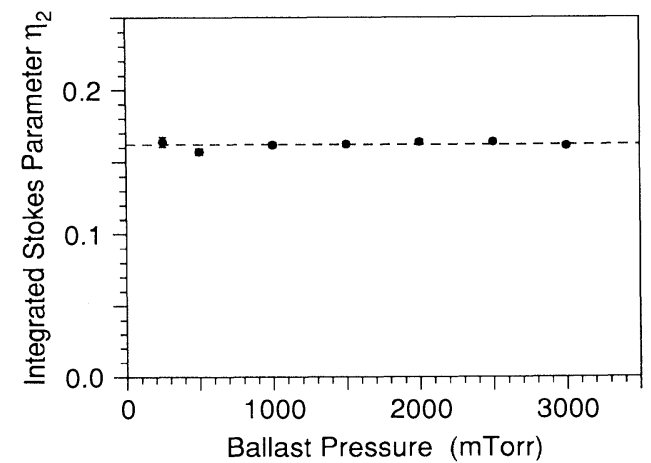

FIG. 6. Pressure dependence of $\eta_{2}$ in neon at $25 \mathrm{eV}$ electron energy. The dashed line represents the weighted mean of all data points, yielding a reduced- $\chi^{2}$ value of $\chi_{v}=1.45$ for six degrees of freedom. A ballast pressure of 1 Torr corresponds to a target density of roughly $10^{10} \mathrm{~cm}^{-3}$. 
age on the lens elements and therefore may alter the measured polarization as the tuning of the electron gun is changed, even when the electron energy in the interaction region is fixed. Such background can occur below the nominal "threshold" voltage, because of higher beam energies upstream in the electron lenses.

(ii) Scattering from the walls of the tube surrounding the interaction region.

(iii) Though we used a narrow-bandpass interference filter, it is possible to detect photons arising from other excited states of the target atoms. While for several of the gases there are lines with similar excitation thresholds within $10 \AA$ of the measured lines, their apparent intensity [26] and the narrow width of the filter essentially eliminated their influence on our measurements. Similarly, contributions due to photons coming from transitions in the $\mathrm{H}_{2}$ and $\mathrm{N}_{2}$ background gas are expected to be nonexistent, except possibly for neon where a minor line in the $\mathrm{N}_{2}$ spectrum has a wavelength of $6411 \AA$, $9 \AA$ from the neon line.

Typical background count rates from sources (i), (ii), and (iii) were less than $45 \mathrm{~s}^{-1}$. To correct for this electron-related background, the count rate was measured $0.5 \mathrm{eV}$ below the excitation threshold and then subtracted from the dark-count-corrected count rate. In this energy region below threshold the electron-relatedbackground contributions were independent of electron energy. This correction made little difference to our results, except within $0.2 \mathrm{eV}$ of threshold where it contributed to large errors and difficulty in accurately identifying the threshold voltage.

\section{RESULTS AND DISCUSSION}

We have measured the Stokes parameters $\eta_{1}, \eta_{2}$, and $\eta_{3}$ from threshold to $100 \mathrm{eV}$ for the $n p^{5}(n+1) p[5 / 2]_{3^{-}}$ $n p^{5}(n+1) s[3 / 2]_{2}$ transitions in neon through xenon and the $4 p^{5} 5 p[5 / 2]_{2}-4 p^{5} 5 s[3 / 2]_{1}$ transition in krypton. The $4 p^{5} 5 p[5 / 2]_{2}$ state in $\mathrm{Kr}$ was chosen because it is strongly intermediately coupled $[\alpha=0.708, \beta=0.684$, and $\delta=-0.173$; Eq. (6) and Ref. [27]] and there is a 0.6$\mathrm{eV}$ energy gap between its threshold for excitation and that of the first state that can cascade into it. These characteristics make it possible to use $\eta_{1}$ for this state as a measure of the importance of internal target spin-orbit effects. The results of these measurements are shown in Figs. 7-10.

Also shown in Figs. 9 and 10 are the results of a semirelativistic first-order distorted-wave Born (DWB1) calculation. This calculation is similar to that reported in Furst et al. [2] except that here we have used the numerical wave functions of Froese-Fischer [28] instead of those of Eissner, Jones, and Nussbaumer [29]. The DWB1 calculation includes relativistic effects for the projectile electron (the basis of Mott scattering) while using a nonrelativistic description of the target-atom wave functions. While the DWB1 results are sensitive to the atomic wave functions, we have found that different wave functions give qualitatively similar results.

At threshold we are able to make predictions of the polarizations as discussed in Sec. II. At higher energies, re-

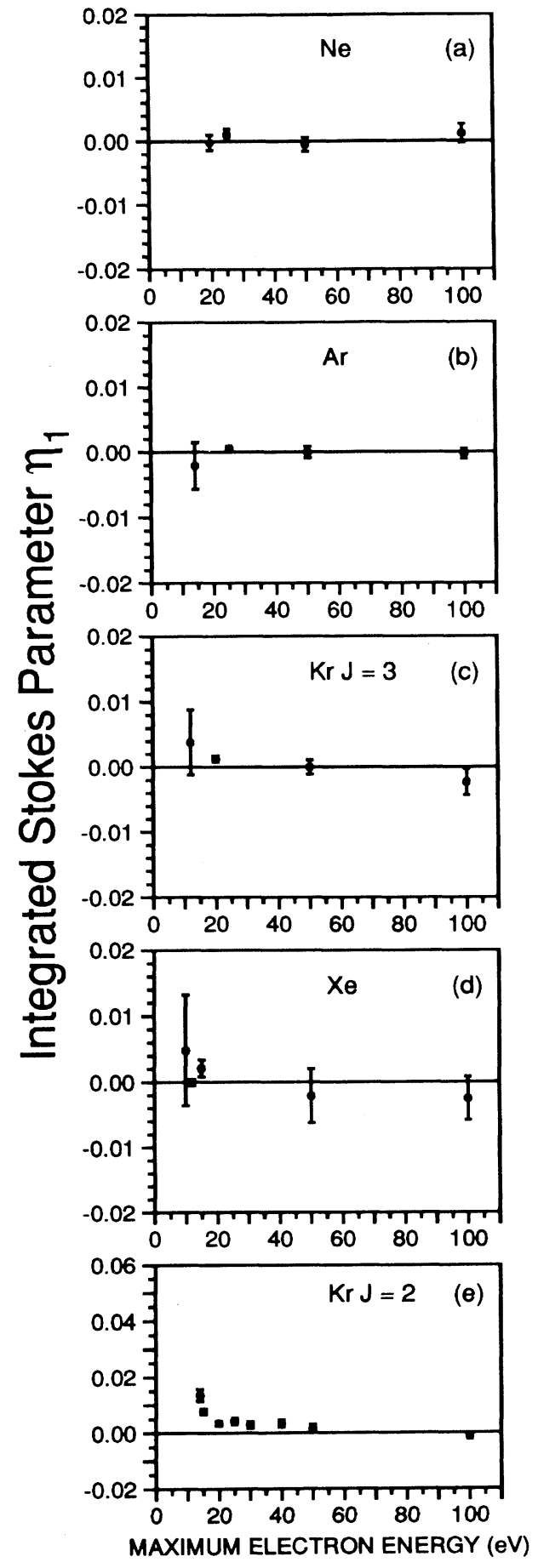

FIG. 7. Integrated Stokes parameter $\eta_{1}$ as a function of maximum electron energy. The high-energy results for the $n p^{5}$ $(n+1) p[5 / 2]_{3}-n p^{5}(n+1) s[3 / 2]_{2}$ transition in the noble gases are shown in (a)-(d). The high-energy results for the $4 p^{5}$ $5 p[5 / 2]_{2}-4 p^{5} 5 s[3 / 2]_{1}$ transition in $\mathrm{Kr}$ are given in (e). Indicated uncertainties in the data are statistical. The results have been corrected for nonideal polarizing element characteristics, but not for the solid angle of observation subtended by the collection lens. The maximum electron energy is approximately 75 $\mathrm{meV}$ greater than the mean electron energy. 


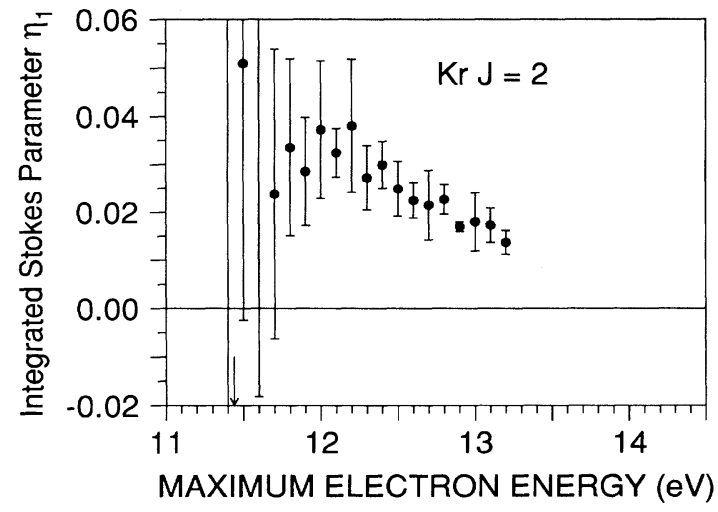

FIG. 8. Near-threshold $\eta_{1}$ results for the $4 p^{5} 5 p[5 / 2]_{2}-4 p^{5}$ $5 s[3 / 2]_{1}$ transition in $\mathrm{Kr}$. cent measurements by Clark [30] indicate that cascade contributions to the total cross section for the $5 p^{5}$ $6 p[5 / 2]_{3}$ state in xenon are about $50 \%$ at $15 \mathrm{eV}$ and that they increase with energy. We expect similar behavior in the other noble gases. Consequently, our results may not be interpreted simply above the first cascade threshold. In the following, we will discuss each Stokes parameter individually, considering especially systematic trends of the data with both beam energy and target $Z$. We will then consider several general physics issues raised by the experimental results.

\section{A. Stokes parameter $\eta_{1}$}

As discussed in Sec. II, $\eta_{1}$ provides a simple test for the importance of spin-orbit forces in the collision. We have
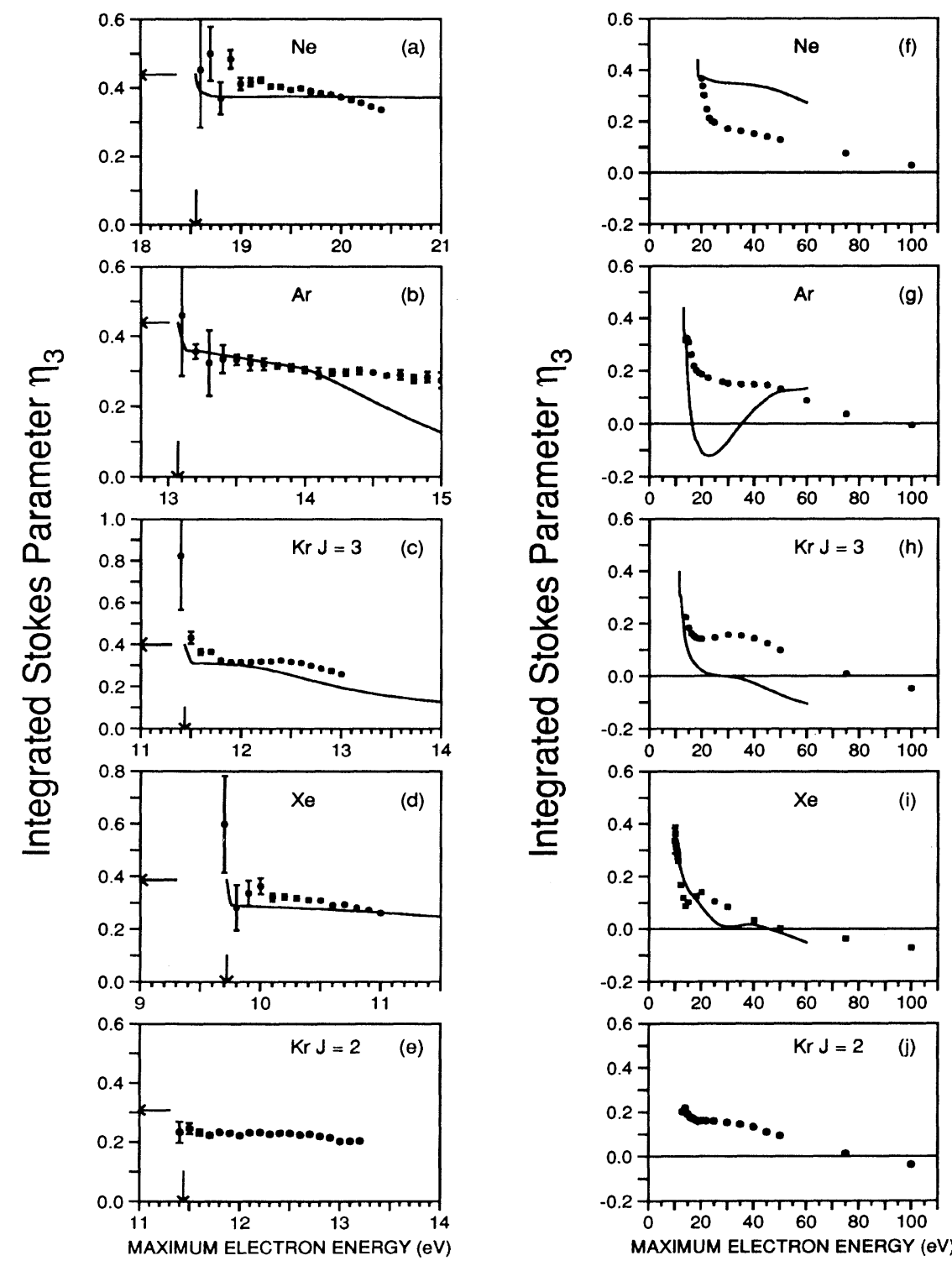

FIG. 9. Integrated Stokes parameter $\eta_{3}$ as a function of maximum energy with the results of the DWB1 calculation indicated by the solid line. The kinematic-polarization limit and excitation threshold energy are indicated by arrows. 
measured $\eta_{1}$ for the pure $L S J=3$ states [see Figs. 7(a) 7(d)] from just below the first cascade excitation threshold to $100 \mathrm{eV}$ and find no evidence that Mott scattering is strong enough to affect the polarization, even with a $\mathrm{Xe}$ target. We recently reported a high-precision measurement of $\eta_{1}$ for $\mathrm{Xe}$ at $9.9 \mathrm{eV}$, just below the first cascade threshold [2]. With full corrections for imperfect polarization optics, our previous value of $\eta_{1}(0.004 \pm 0.006)$ at $9.9 \mathrm{eV}$ is revised to $0.005 \pm 0.008$. Thus, the only dynamical spin effect that is contributing to the integrated collision process for the $J=3$ states is exchange. It may be possible to do such a measurement using heavier targets such as mercury [2], where Mott scattering should be more pronounced.

At energies above the first cascade threshold, one might expect departures from zero due to the contributions of non-well- $L S$-coupled states to the population of the radiating state. However, the high-energy results in- dicate that this is not the case. Certainly the nexthigher-lying state, generally a $n p^{5} n d{ }^{3} F_{4}$ state which can only decay into the ${ }^{3} D_{3}$ level, is a Russell-Saunders state, as are the $n p^{5}(n+2) s^{3} P_{2}$ states, all of which will cascade strongly into the ${ }^{3} D_{3}$ level.

The situation for the $J=2$ state in krypton is much different [Figs. 7(e) and 8]. Near threshold, $\eta_{1}$ has definite nonzero values. Since our results for $\eta_{1}$ for the $J=3$ state are consistent with zero, indicating that spinorbit coupling to the continuum electron during the collision is negligible for these systems, the nonzero $\eta_{1}$ 's for the $J=2$ state, which is in the same manifold, represent an unambiguous signature of the effect of the breakdown of $L S$ coupling on the fluorescence polarization. This is consistent with the strong mixing indicated by the intermediate-coupling coefficients.

In collisions of this type, it is not necessary to view the off- $z$-axis alignment $\left(\left\langle\mathfrak{J}_{21}^{\dagger}(J)\right\rangle_{P}\right)$ as resulting from mag-
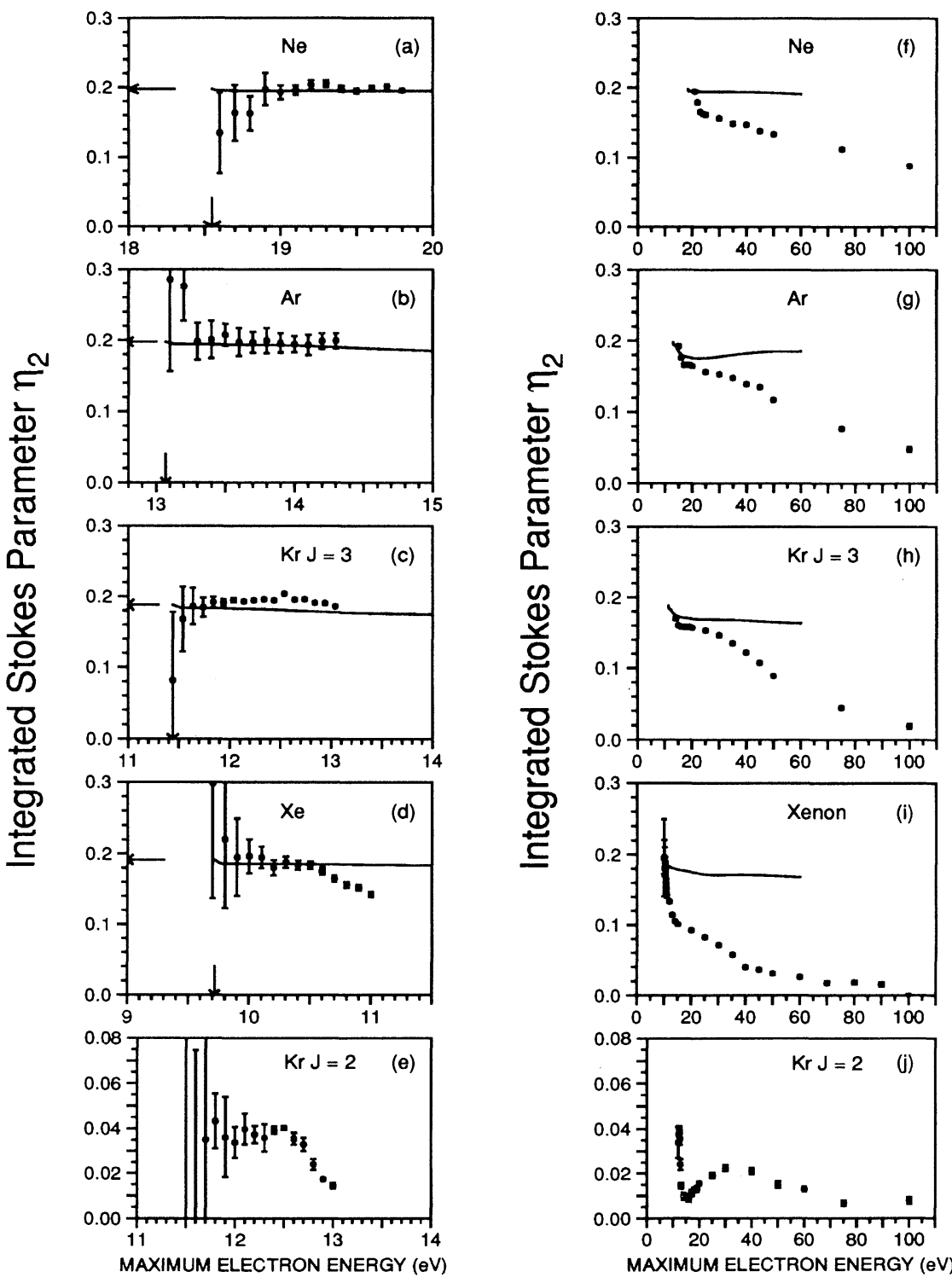

FIG. 10. Integrated Stokes parameter $\eta_{2}$ as a function of maximum energy. The DWB1 calculation normalized to an electron polarization of $P_{e}=0.27$ is indicated by the solid line. The kinematically determined threshold for the same electron polarization and the excitation threshold for each state are indicated by arrows. 
netic spin flips occurring during the collision. Instead, it is as a result of the "relaxation" of the atom into its finestructure eigenstate, which is not $L S$ coupled. One can envision a final trajectory-averaged charge cloud with only axial alignment but with spin orientation along $\hat{\mathbf{y}} \mathrm{im}$ mediately following the collision. Over the fine-structure relaxation time, however, the charge cloud "tilts" off the $z$-axis in the $x-z$ plane, where it then remains fixed in space until it emits a photon. This conversion of orientation along $\hat{\mathbf{y}}$ to off-axial alignment is the result of the strong magnetic intra-atomic forces responsible for the breakdown of $L S$ coupling in the excited state; a pure Russell-Saunders state does not tilt away from the $z$ axis.

\section{B. Stokes parameter $\eta_{3}$}

It can be seen in Figs. 9(a)-9(d) that at threshold our measured $\eta_{3}$ polarizations for the $J=3$ states are consistent with the threshold predictions discussed earlier. We see that, except for possibly neon, the polarization drops away from the threshold value and then remains constant or decreases slowly for another volt after threshold. There is then a general decrease for several electron volts after threshold and then either a slight increase or a plateau before a slow, monotonic decrease. These measurements have some similarities with previously measured helium polarizations [31]. Near threshold though, the ${ }^{3} D_{3}$ results look very different from, e.g,. the measurements of Heddle, Keesing, and Parkin [31] for the $3^{3} P$ to $2{ }^{3} S$ transition in helium. In helium there is a rapid decrease in the polarization to near zero within a few tenths of an electron volt of threshold. Low values of $\eta_{3}$ persist for several electron volts, even after cascades begin to have an influence. The noble-gas results show some tendency for a rapid decrease near threshold, but very quickly stabilize to a value within $25 \%$ of the threshold prediction, with no structure to indicate the effect of resonances on our measurements.

There has been much speculation on the possible causes of the depolarization measured near threshold for several lines in helium. It has been suggested that the sudden decrease in linear polarization $\eta_{3}$ near threshold for the $3{ }^{3} P$ to $2{ }^{3} S$ transition in helium may be due to the presence of a variety of negative-ion resonances that strongly affect the population of the excited state [32]. Since $\eta_{3}$ for all the $J=3$ states and the $J=2$ state in krypton has no structure near threshold even in the presence of what are, in general, strong resonances [33], it would appear that the resonances do not decay into either state. If resonances were to have an effect similar to that in helium, then we would expect that there would be a more dramatic decrease in polarization than we observe even with the present energy resolution, since we have previously observed the sudden, large decrease in $\eta_{3}$ for the $3{ }^{3} P-2{ }^{3} S$ transition in helium [34].

In the near-threshold region, the DWB1 calculation has surprisingly good agreement with the measurements. Though kinematically constrained to agree with the $L S$ threshold prediction if $L S$ coupling for the system holds, it can be seen that for all the $J=3$ states it drops rapidly in less than $0.15 \mathrm{eV}$ and then remains quite close to the experimental values over the energy range unaffected by cascades.

Although at higher energies our measurements are significantly affected by cascades, several general features should be noted. For the $J=3$ states all of the gases exhibit a rapid decrease in $\eta_{3}$ for many volts after threshold, and then the change becomes less rapid. In xenon and krypton there are definite minima at 14 and $19 \mathrm{eV}$, respectively. Neon and argon do not show minima at corresponding energies but, instead, pronounced changes in slope. As one goes from neon to xenon, the changes in slope become larger and occur at lower energies. This energy dependence is probably associated with a lowering of the threshold energy and a decreasing energy gap between levels that occur as the target atoms get heavier. At yet higher energies all the measurements show a tendency towards negative values. Only in neon is $\eta_{3}$ greater than zero at $100 \mathrm{eV}$, and it becomes increasingly more negative as the atoms get heavier. This is consistent with the kinematic requirement that only $M_{L}=|L|$ states are populated at infinite energy, and therefore $\eta_{3}$ must be negative [2], even when strongly influenced by contributions from cascading transitions. (The upper-lying cascade states must obey the same kinematic rule.)

With possibly more than a $50 \%$ contribution from cascades, the DWB1 would not be expected to agree with the high-energy $\eta_{3}$ measurements. However, except for neon, the theory indicates the rapid decrease observed in the first $10 \mathrm{eV}$ after the excitation threshold. The DWB1 calculations also show distinct changes in slope for Ar and $\mathrm{Kr} J=3$ states at energies close to similar changes in the measured values. It would appear that at these energies pure ${ }^{3} D_{3}$ excitation is still significant.

As would be expected the krypton $J=2$ near-threshold values [Fig. 9(e)] show no trend towards the $L S$-coupled threshold predictions. There is no structure and the high-energy results [Fig. 9(j)] show changes in the slope in a similar energy range to the krypton $J=3$ results. Again, $\eta_{3}$ tends to a negative value at high energies, as it must.

\section{Stokes parameter $\boldsymbol{\eta}_{\mathbf{2}}$}

The $\eta_{2}$ results are shown in Figs. 10a(a)-10(j). We indicate on the graphs the predicted threshold $\eta_{2}$ values for each gas assuming an electron polarization of $27 \%$. It should be pointed out that these measurements were taken over several months with several cathode activations, so $27 \%$ represents an estimate of the average electron polarization over that time. In all of the $J=3$ results we see excellent agreement with the threshold predictions and the DWB1 calculation for up to an electron volt above the threshold energy. The sustained agreement with both the threshold value and the DWB1 is due to the fact $\eta_{2}$ is only slightly affected by the alignment term $\eta_{3}$ which in turn changes very slowly in this energy range. The mechanism dominating the value of $\eta_{2}$ is the transfer of spinangular momentum by exchange excitation, with only a small decrease due to the induced alignment.

It is interesting to note that for energies greater than a few electron volts above threshold, $\eta_{2}$ shows distinct changes in slope at the same energies as do the $\eta_{3}$ mea- 
surements. We have shown that for well- $L S$-coupled states, there is a simple relationship between $\eta_{2}$ and $\eta_{3}$ [Eq. (7)], so these similarities are not unexpected, indirect as they may be in an energy regime dominated by cascading. As the targets become heavier and the energy higher, $\eta_{2}$ tends towards smaller values, though in the energy range measured only the value for xenon actually drops to zero. Based on the high-energy predictions (Table II), we would expect $\eta_{2}$ to drop by a relatively small amount over this range. However, cascades from intermediately coupled "triplet" and "singlet" states will contain contributions from direct scattering processes which transfer no orientation into the atomic system. The net result is that $\eta_{2}$ must go to zero at high energies where direct scattering dominates the scattering process.

The krypton $J=2 L S$-coupling threshold prediction [Fig. 10(e)] is zero, but the present results have too large an error near threshold to judge how well they agree with this prediction. Just a few tenths of a volt above threshold, however, $\eta_{2}$ is nonzero and maintains a steady value for almost another volt even after the onset of cascades. Based on the lack of strong energy dependence near threshold for $\eta_{2}$ in the $J=3$ states, it is unlikely that the $J=2$ data suddenly fall to the predicted $L S$-coupling value of zero at threshold. This significant departure from the $L S$-coupled threshold prediction is of course consistent with the departure from zero of $\eta_{1}$ for this state. At higher energies [Fig. 10(j)], $\eta_{2}$ shows a more pronounced dip than any of the $J=3$ states; however, the reason for this is unclear.

\section{CONCLUSIONS}

In conclusion, we have presented measurements of integrated Stokes parameters involving well- $L S$-coupled states in the noble gases. Together with a measurement of a non-well- $L S$-coupled state, we have demonstrated the effectiveness of using $\eta_{1}$ to prove spin-orbit effects unimpeded by other dynamical or kinematic considerations. The zero value of $\eta_{1}$ for all the $J=3$ transitions demonstrates that continuum spin-orbit coupling has a negligible influence in collisions of this type when integrated over all scattering angles. This is quite different from differential elastic-scattering experiments where quite large asymmetries due to Mott scattering have been observed [35] and where the asymmetry, integrated over scattering angle, is typically of the order of at least several percent. The difference between elastic and inelastic collisions in terms of producing scattering asymmetries has been discussed by Hanne [36]. Hanne's assertion that elastic scattering should, in general, lead to larger Mott scattering effects than does inelastic scattering would appear to be borne out by comparing our results with the integrated values of Garcia-Rosales, Müller, and Kessler [35]. We note, however, the counterexample of elastic versus inelastic scattering from $\mathbf{H g}$ (see, e.g., Refs. [21] and [37]).

Because Mott scattering, when integrated over all scattering angles, has a negligible effect on the photon polarization in the collisions we have studied, Eq. (7) describes the relationship between $\eta_{2}, \eta_{3}$, and $P_{e}$ accurately below the excitation threshold energy for the first cascade transition. In addition, the slowly varying nature of $\eta_{3}$ after the initial drop near threshold means that an electron-polarization measurement will not be as sensitive to the electron-energy and energy distribution as would measurements involving helium. Thus the ${ }^{3} D_{3}$ state in any of these gases may be suitable for use in electronpolarization measurements. Further studies of these systems to check their worth for electron polarimetry seem warranted.

An integrated $\eta_{1}$ measurement may seem to be an insensitive indicator of spin-orbit effects. Its virtue is that a nonzero value arising from a pure Russell-Saunders state is an unambiguous sign of inelastic Mott scattering. In differential-scattering experiments involving the excitation of definite $J$ states, both the "fine-structure" effect and Mott scattering may cause more electrons to be scattered to one side than the other. When such scattering asymmetries are observed after scattering from atoms heavy enough for relativistic effects to be important, it is impossible to distinguish between the two phenomena. However, if the excited state is well- $L S$-coupled, and a non-zero $\eta_{1}$ attributable to the breakdown of the Percival-Seaton hypothesis is measured, then only Mott scattering can cause an integrated nonaxial alignment, $\left\langle\mathfrak{\Im}_{21}^{\dagger}(J)\right\rangle_{P}$. The fine-structure effect arises solely due to orientation induced by exchange, $\left\langle\mathfrak{\Im}_{11}(J)\right\rangle_{P}$, so that in an integrated measurement its influence is seen only in $\eta_{2}$ measurements and may be completely described assuming $L S$ coupling.

It is also worthwhile to note that $\eta_{1}$ has many similarities to the parameter $\rho_{00}^{n}$ that is used to describe the effect of spin-orbit forces in electron-photon coincidence experiments with unpolarized electrons [38]. Departure from the $L S$-coupled predictions for both parameters indicates the importance of spin-orbit effects. In a manner similar to $\eta_{1}$, if a pure $L S$-coupled state is excited, a departure of $\rho_{00}^{n}(J)$ from the $L S$-coupled prediction may be used as an indication of the influence of Mott scattering on the scattering process. There are several differences though, the most important being that a $\rho_{00}^{n}(J)$ due to Mott scattering can be observed without using polarized electrons. Another significant difference is that two Stokes parameters, one for photon emission in the scattering plane and the other for emission out of the plane, are required to determine $\rho_{00}^{n}$. This topic will be discussed further in an upcoming paper [39].

Another surprising feature of our measurements is the complete lack of evidence for effects due to negative-ion resonances. This is quite different from previous helium $\eta_{3}$ measurements which seem to be strongly affected by resonances. Integrated measurements of $\eta_{1}, \eta_{2}$, and $\eta_{3}$ for the excitation of $\mathrm{Hg}{ }^{3} P_{1}$ states are also strongly influenced by resonances [3]. Indeed, even with our poor energy resolution, we have seen the sudden decrease in polarization for the $\mathrm{He} 3{ }^{3} \mathrm{P}$ to $2{ }^{3} \mathrm{~S}$ transition.

\section{ACKNOWLEDGMENTS}

This work was supported by National Science Foundation Grants Nos. PHY-9007721, PHY-9014103, and 
PHY-8813799. We thank D. Cartwright, G. Csanak, and J. Abdallah of Los Alamos National Laboratory for providing intermediate-coupling coefficients for the $n p^{5}$ $(n+1) p$ levels in the noble gases and for useful discussions. We would also like to thank J. D. Clark of Wright
State University for providing data on the relevant cascading transitions prior to publication. In addition, we thank J. Nickel, J. W. McConkey, A. Stauffer, R. McEachran, S. Trajmar, and M. A. M. Khakoo for useful discussions.
*Present address: Centre for Atomic, Molecular and Surface Physics, Physics Department, The University of Western Australia, Nedlands, Perth 6009, Australia.

[1] K. Blum, Fundamental Processes in Energetic Atomic Collisions, edited by H. O. Lutz, J.S. Briggs, and H. Kleinpoppen (Plenum, New York, 1983), p. 651.

[2] J. E. Furst, T. J. Gay, W. M. K. P. Wijayaratna, K. Bartschat, H. Geesmann, M. A. Khakoo, and D. H. Madison, J. Phys. B 25, 1089 (1992).

[3] A. Wolke, K. Bartschat, K. Blum, H. Borgmann, G. F. Hanne, and J. Kessler, J. Phys. B 16, 639 (1983).

[4] F. Eschen, G. F. Hanne, K. Jost, and J. Kessler, J. Phys. B 22, L455 (1989).

[5] P. Na $\beta$, M. Eller, N. Ludwig, E. Reichert, and M. Webersinke, Z. Phys. D 11, 71 (1989).

[6] K. Blum and K. Bartschat, Z. Phys. A 304, 85 (1982).

[7] K. Blum, Density Matrix Theory (Plenum, New York, 1981).

[8] I. C. Percival and M. J. Seaton, Philos. Trans. R. Soc. London Ser. A 251, 113 (1958).

[9] P. S. Farago and J. S. Wykes, J. Phys. B 2, 747 (1969).

[10] M. Eminyan and G. Lampel, Phys. Rev. Lett. 45, 1117 (1980).

[11] M. Uhrig, A. Beck, J. Goeke, F. Eschen, M. Sohn, G. F. Hanne, K. Jost, and J. Kessler, Rev. Sci. Instrum. 60, 872 (1989).

[12] T. J. Gay, J. Phys. B 16, L553 (1983).

[13] K. Bartschat, K. Blum, G. F. Hanne, and J. Kessler, J. Phys. B 14, 3761 (1981).

[14] CRC Handbook of Chemistry and Physics, 61st ed., edited by Robert C. Weast (CRC Press, Boca Raton, 1981).

[15] T. J. Gay, M. A. Khakoo, J. A. Brand, J. E. Furst, W. V. Meyer, and W. M. K. P. Wijayaratna, Rev. Sci. Instrum. 63, 114 (1992).

[16] D. T. Pierce, R. J. Celotta, G.-C. Wang, W. N. Unertl, A. Galejs, C. E. Kuyatt, and S. R. Mieclczarek, Rev. Sci. Instrum. 51, 478 (1986).

[17] F.-C. Tang, M. S. Lubell, K. Rubin, A. Vasilakis, M. Eminyan, and J. Slevin, Rev. Sci. Instrum. 57, 3004 (1986).

[18] X. Q. Guo, D. M. Crowe, M. S. Lubell, F. C. Tang, A. Vasilakis, M. Eminyan, and J. Slevin, Rev. Sci. Instrum. 61, 1858 (1990).
[19] L. A. Hodge, T. J. Moravec, F. B. Dunning, and G. K. Walters, Rev. Sci. Instrum. 50, 5 (1979).

[20] D. M. Campbell, C. Hermann, G. Lampel, and R. Owen, J. Phys. E 18, 663 (1985).

[21] J. Kessler, Polarized Electrons, 2nd ed. (Springer-Verlag, Berlin, 1985), p. 230.

[22] T. J. Gay, J. A. Brand, M. C. Fritts, J. E. Furst, M. A. Khakoo, E. R. Mell, M. T. Sieger, and W. M. K. P. Wijayaratna (unpublished).

[23] C. B. Lucas, Vacuum 23, 395 (1973).

[24] D. W. O. Heddle, Electrostatic Lens Systems (Hilger, Bristol, 1991).

[25] V. J. Ehlers and A. Gallagher, Phys. Rev. A 7, 1573 (1973).

[26] A. R. Striganov and N. S. Sventitskii, Tables of Spectral Lines of Neutral and Ionized Atoms (IFI/Plenum, New York, 1968).

[27] D. Cartwright, G. Csanak, and J. Abdallah (private communication).

[28] C. Froese-Fischer, Comput. Phys. Commun. 4, 107 (1972).

[29] W. Eissner, M. Jones, and H. Nussbaumer, Comput. Phys. Commun. 8, 270 (1974).

[30] J. D. Clark (private communication).

[31] D. W. Heddle, R. G. W. Keesing, and A. Parkin, Proc. R. Soc. London, Ser. A 352, 419 (1977).

[32] U. Fano and J. Macek, Rev. Mod. Phys. 45, 553 (1973).

[33] S. J. Buckman, P. Hammond, G. C. King, and F. H. Read, J. Phys. B 16, 4219 (1983).

[34] T. J. Gay, J. E. Furst, H. Geesmann, M. A. Khakoo, D. H. Madison, W. M. K. P. Wijayaratna, and K. Bartschat, Correlations and Polarizations in Electronic and Atomic Collisions and $(e, 2 e)$ Reactions, edited by P. J. O. Teubner and E. Weigold (Institute of Physics, Bristol, 1992), p. 265.

[35] C. Garcia-Rosales, H. Müller, and J. Kessler, J. Phys. B 21, L477 (1988).

[36] G. F. Hanne, Phys. Rep. 95, 97 (1983).

[37] D. H. Madison and W. N. Shelton, Phys. Rev. A 7, 514 (1973).

[38] N. Andersen, J. W. Gallagher, and I. V. Hertel, Phys. Rep. 165, 1 (1988).

[39] J. E. Furst, D. H. Madison, and T. J. Gay (unpublished). 